\title{
Application of Agent-Based Modelling AND Simulation TOOLS
}

\author{
RAKIC, K.
}

Abstract: Simulation is experimenting with an imitation, or model, of the observed system, and observing its behaviour over time, with the purpose of better understanding and/or improving the system. If the system is inaccessible, dangerous or unacceptable for inclusion in the research, if it is designed but not yet built, abstract or just does not exist, system simulation is often the best option for researching such systems. Elements of these models can be implemented as software entities that perceive their environment and respond autonomously to stimulation. We call these entities intelligent agents. Software tools that enable the exploration of complex natural, social, and technical phenomena and systems are called Agent-Based Modelling and Simulation tools. This paper provides an overview of the wide range of possible applications of these modern computer tools.

Key words: simulation, modelling, intelligent agents, agent-based modelling and simulation tools
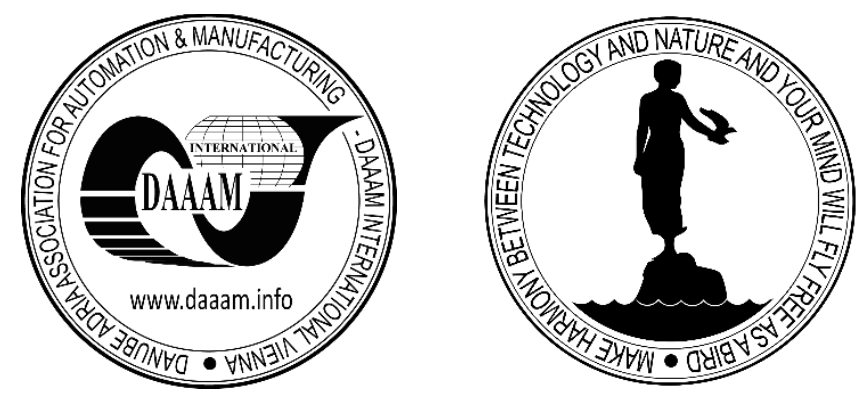

Authors' data: Dr. Sc. Rakic, K[resimir], University of Mostar, Faculty of Mechanical Engineering, Computing and Electrical Engineering, Matice hrvatske b.b., 88000, Mostar, Bosnia and Harzegovina, kresimir.rakic@ fsre.sum.ba

This Publication has to be referred as: Rakic, K[resimir] (2021). Application of Agent-Based Modelling and Simulation Tools, Chapter 17 in DAAAM International Scientific Book 2021, pp.213-222, B. Katalinic (Ed.), Published by DAAAM International, ISBN 978-3-902734-31-0, ISSN 1726-9687, Vienna, Austria DOI: $10.2507 /$ daaam.scibook.2021.17 


\section{Introduction}

The rapid development of information and communication technology (ICT) in recent times has given the wider scientific community the opportunity to approach problems in hitherto unimaginable ways. Many natural, social and technical phenomena and systems are difficult to access or completely inaccessible. They can be dangerous to observe and explore or abstract and invisible to the human eye.

Using modern ICT tools, it is possible to model and simulate such phenomena and systems and manipulate them with the aim of analysing the system and studying its functioning. Elements of these systems can be implemented as software entities that perceive their environment and respond autonomously to stimulation. This description largely coincides with the standard definition of an intelligent agent. Therefore, this paper deals with agent-based modelling and simulation tools (ABMS).

In this paper, we will define the basic concepts of simulation, modelling and intelligent agents whose synergy has realized ABMS tools. Today, their application can be found today in almost every field of professional and scientific research.

\section{Definition and Basic Features of Simulation}

In everyday life, we often encounter the concept of simulation. Weather forecasters regularly show us simulations of the weather in the days that follow. Children, and sometimes adults, play with physical models of cars, railways, ships, and, more recently, aircraft that, remotely controlled, simulate the behaviour of real vehicles. Numerous computer applications simulate a whole range of human activities, which allows us to test our skills as drivers, researchers, urban planners and the like.

From a historical point of view, simulations used in different areas of human action have evolved largely independently. Nevertheless, research in the field of cybernetics and systems theory during the 20th century, combined with the increasing application of computers in all these fields, led to a certain unification and systematic presentation of the concept of simulation.

Simulation is today an increasingly present methodology for solving problems from different areas of human activity. It is used to describe and analyse the behaviour of the system, to detect causal relationships within the system, but also as an auxiliary tool in shaping real systems, both existing and conceptual.

In its most general sense, simulation can be defined as an imitation of a system. Imitation means imitating or copying something else. For example, a counterfeiter imitates the work of a great artist or seeks to faithfully copy monetary denominations. For an athlete who acts as if an offence has been committed on him, although this is not the case, we say he is simulating. Computer-Aided Design systems (CAD systems) allow the imitation of the design of physical artefacts or entire production facilities. However, the key difference between these imitations and the previously described examples is that they do not involve the passage of time.

There is, therefore, a difference between the concept of static simulation that mimics a system at a particular point in time and the concept of dynamic simulation that includes time course (Law \& Kelton, 2000). 
The term simulation is mainly used in the context of dynamic simulation, so we can define it as an imitation of the operation of a real-world process or system over time (Banks et al., 2001).

We will further analyse certain aspects of this definition. We will first clarify the notion of a system. In general, a system is a set of components, organized for a purpose (Coyle, 1996). We distinguish four main categories of systems (Checkland, 1981):

- Natural systems are systems whose origin is related to the nature around us (e.g., atom, time system of the planet Earth, solar system, etc.).

- Artificial physical systems are systems created as a result of human action (e.g., house, car, production plant, etc.).

- Artificial abstract systems are those systems that are the result of human action, (e.g., mathematics, literature, etc.).

- Systems of human activity are systems of consciously or unconsciously arranged activities of people (e.g., family, city, political system, etc.).

In practice, it is possible to mimic the operation of all these types of systems, i.e., to simulate them. Simulating a real system first requires the development of a system model. The system model represents the key characteristics, behaviours, and functions of the selected physical or abstract system or process. The model, therefore, represents the system itself, while the simulation represents the functioning of the system over time.

Another aspect of the definition that needs to be further explored is to consider the purpose of simulation models. In his general discussion of models, Pidd identifies the need to understand, change, manage, and control reality as the purpose of model building (Pidd, 2009). Speaking of simulation models, their purpose is to better understand the system and identify possible improvements to the system. A better understanding of the system, as well as the identification of possible improvements, are important for future decision-making in the actual system.

Another important feature of the model is the emphasis on simplification. Namely, the system model rarely includes all aspects of the system but concentrates not on those that are crucial for the functioning of the system. Even if it is possible to cover all the details, this is probably not desirable, as it would require too much time to gather and incorporate into the model all aspects of the system.

The purpose of modelling the system should be considered when deciding on the application of simulation. A simulation is an experimental approach to modelling in which the user of the simulation changes the inputs of the system, i.e., explores alternative scenarios, and observes the behaviour of the model until he gains enough understanding of its work and identifies ways to improve the real system. For example, it is possible to predict the average waiting time for telephone users in a call centre when several operators are employed, and the task of the person using the simulation model is to change the input (operator number) and observe the simulation model to determine the effect of the change.

With the additionally described essential aspects of simulation, it is possible to more precisely define simulation as experimenting with a simplified (computer) imitation of the observed system and observing its behaviour over time, with the purpose of better understanding and/or improving the system. 


\section{When to Apply the Simulation?}

When deciding on the possible application of a simulation, it is necessary to determine whether this approach is the most appropriate to the problem we are investigating. For this purpose, it is necessary to consider the characteristics of the observed system and determine the advantages and disadvantages of simulation compared to other methods of system analysis.

\subsection{System Characteristics}

Many real systems are subject to variability. These can be predictable (e.g., changing the number of operators in the call centre during the day to meet a variable number of customer calls or planned interruptions in the production facility) or unpredictable changes (e.g., the number of patients arriving at an emergency hospital or dismantling equipment in a flexible production cell). Both forms of variability are present in most operating systems.

The systems are also interconnected, i.e., their components do not function in isolation, but affect each other by their action. In doing so, a change in one part of the system leads to a change in another part of the system. The effects of interconnections in the system are difficult to predict correctly, especially when variability is present.

The interconnections of individual components within a system are often not oneway, especially in complex systems. In such systems, we often encounter loop structures or feedback that may involve multiple system components. In doing so, physical objects and information often travel in opposite directions, making system behaviour even more complex and more difficult to predict.

Thus, most real systems are inherently variable, interconnected, and complex. It is difficult to predict the behaviour of systems that are subject to either variability, interconnection, or complexity, and it is almost impossible to do the same for systems that are potentially subject to all the above. Simulation models, however, can explicitly represent the variability, interconnectedness, and complexity of a system. As a result, it is possible to predict system performance by simulation, compare alternative performances as well as determine the effect of alternative rules on system performance. Therefore, simulation is often a convenient approach to system modelling.

\subsection{Advantages of Simulation}

Simulation is not the only method of analysis and research of real systems to get to know and improve them. It is possible to experiment with a real system or use some other system modelling approach. We will list the advantages that simulation has over alternative approaches.

Instead of developing and using a simulation model, experiments can also be conducted on a real system (e.g., additional check-in counters at the airport, etc.). The advantages that simulation has over direct experimentation with a real system include (Robinson, 2004): 
- Lower costs. Experimenting with a real system is potentially expensive due to the need to break established procedures and try new ideas. Additionally, if the changes are not positive, system user dissatisfaction may occur.

- Shorter experimentation time. Direct experimentation is time consuming and can take a longer time, while simulation can run faster than real time, allowing exploring many ideas in a shorter period.

- Ability to control experimental conditions. When researching the effect of alternative ideas, it is important to control the conditions in which the experiment is performed, so that their effect can be directly compared. This is difficult, sometimes even impossible, to achieve when we experiment with a real system, and simply in the case of experimenting with a simulation model.

- In a situation where a real system does not yet exist, direct experimentation is not possible. The only alternative is to develop a credible system model.

As noted earlier, simulation is not the only model used to better understand and improve real-world systems. Other modelling approaches include simple and complex calculations in the form of systems of mathematical equations, spreadsheets, more complex mathematical and heuristic methods (linear programming, dynamic programming, genetic algorithms, etc.). The advantages of simulation over other modelling methods are primarily due to the simpler modelling of system variability and the effect that variability causes. Most alternative methods of modelling real systems are not able to consider the natural variability of the real system it describes, and with methods that can adapt to the changing nature of the described system, such adaptation significantly increases their complexity. Another significant advantage of the simulation is the fact that it does not require restrictive baseline system assumptions or requires them to a minimal extent because of the tendency to simplify the model or as a substitute for missing data.

On the other hand, many other approaches to modelling strictly require the initial assumptions they need to work successfully. Finally, the transparency of the simulation should be added. Compared to other models of real systems modelling, it is more intuitive, and it can often create an animated representation of the system, making it more credible and reliable, especially for users who are not experts in the modelling field.

\subsection{Disadvantages of Simulation}

Of course, there are also cases when another approach to system modelling is appropriate and satisfactory, i.e., cases in which simulation is not necessary or appropriate.

Several disadvantages of this approach should be considered when using simulations. The costs of development and use of the simulation model, which can be significant, should not be neglected. In addition, the simulation is not a quick solution but a time-consuming approach, which further increases the cost of its use.

Most simulation models require a significant amount of data that is not always available and sometimes it takes a long time to transform it into a form that is suitable for simulation. Simulation modelling requires skills and competencies of conceptual 
modelling, validation and statistics, and in some applications, human and project management skills, which are not always readily available.

Also, the simulation can be overly convincing due to the use of an animated, realistic representation of the system, which can further strengthen the tendency to accept the results obtained by computer processing uncritically. Therefore, when interpreting the results obtained by the simulation, it is necessary to consider the validity of the basic model, assumptions and simplifications made.

Due to all the above disadvantages of the simulation approach, it is recommended that it be used when other, simpler, faster and cheaper, methods do not give a satisfactory result (Pidd, 2004).

\section{Areas of Application of Simulation}

The field of application of the simulation encompasses various contexts in which there is a need to optimize the performance of the observed systems. They are extremely useful in queue systems in which entities are processed through a series of stages, with queues being formed between each stage if the system does not have sufficient processing capacity. Many systems can be represented as queuing systems, and the entities that move through the system can be people, physical objects, or information. Queues can describe production systems, transport systems, health care systems, natural resource systems, business process management, computer system performance, and the like.

Simulation is also used to model natural and social systems, to gain insight into their functioning, examine and demonstrate the effectiveness of alternative conditions or modes of action. Simulation is also used in situations where the real system cannot be included in the research, due to its unavailability, dangerous or unacceptable inclusion, the fact that the system is designed but not yet built, or in situations where the system is abstract or simply does not exist (Sokolowski \& Banks, 2009).

Key issues in the simulation include obtaining valid information on the key characteristics and behaviour of system elements about the appropriate shape, using approximations and assumptions for easier system modelling, and checking the accuracy and validity of the simulation outcomes. Procedures and protocols for model verification and validation are a continuous area of academic research and development of simulation technology and practice, especially in the field of computer simulation.

\section{Intelligent Agents and Multi-Agent Systems}

An agent is a physical and/or virtual system that perceives its environment by various sensors and acts on it through its actuators. Agents work in their environment and can include people, robots, computer programs, but also some simple gadgets.

The software agent is a persistent, goal-oriented computer program that responds to its environment, works without continuous direct surveillance and performs certain functions for the end-user or other computer program in a particular environment. Such independent action implies the power to choose a suitable action in a particular situation if it exists. The agent is inhibited by other processes and agents, but also can 
be able to learn from its experience of working in the environment over a long period (Rakic et al., 2020).

Software agents represent an evolutionary step in comparison to conventional computer programs. They can be self-activated and run and they do not require input or interaction with a human user. Software agents can also start, monitor, and shut down other programs or agents.

All agents are computer programs, but not all programs are agents. Key features that differentiate agents from arbitrary programs are, according to Franklin and Graesser, reaction to the environment, autonomy, goal orientation and persistence (Franklin \& Graesser, 1996).

Thus, an agent is an entity that we can define through its perception of the environment in which it is located, by the built-in sensors, and acting in such an environment and on that environment through actuators.

The agent's perception sequence is a complete history of everything that the agent ever perceive. The agent's choice of action at any time may depend only on the last perception or on the entire perception sequence it percept so far. In mathematical terms, we can say that agent behaviour is described by an agent function that maps every perception into the corresponding action.

Russel and Norvig listed five basic types of agents characterized by a degree of expressed intelligent behaviour and ability (Russel \& Norvig, 2000):

- Simple Reflex Agents that select action based on a current observation, ignoring the history of observation.

- Model-Based Reflex Agents that select the action based on the history of observation.

- Goal-Based Agents who choose the action to achieve a specific goal.

- Utility-Based Agents that select the action that will achieve the maximum degree of success.

- All these agents can also be Learning Agents who analyse the experience to choose the action.

Systems in which intelligent agents work, as well as multi-agent systems, are examples of abstract systems. The study of these systems is hardly feasible at the theoretical level. A particular difficulty is the prediction of system behaviour in different situations that the intelligent agent, or several agents in a multi-agent system, perceives by its sensors, and responds to them depending on the defined causal rules. As such, these systems are very suitable for studying and researching using simulation models.

\section{Agent-Based Modelling and Simulation Tools and their Areas of Application}

Widespread use of computer agents that, with ubiquitous digitization, slowly penetrate all spheres of life also caused the need for investigating the behaviour of the systems in which individual agents or groups of agents act autonomously. The number of environments that enable modelling and simulation of such agent-based systems is 
constantly increasing (Rakic, 2020). These solutions are referred as Agent-Based Modelling and Simulation (ABMS) tools.

This motivated numerous authors to conduct screening of available tools, focusing on different aspects of system modelling and simulation (Serenko \& Detlor, 2002; Tobias \& Hofmann, 2004; Railsback et al., 2006; Castle \& Crooks, 2006; Nikolai \& Madey, 2009; Allan, 2010; Abar et al., 2017).

In the most comprehensive survey of ABMS tools, Abar et al. reviewed 85 existing tools. The authors present a concise characterization of existing tools, including the underlying programming language, the type of agent that the system supports, the complexity of the end user system, and the domain for which the system is primarily intended, highlighting their advantages and disadvantages. In this way, they provide a useful reference for engineers, researchers, students, and teaching staff for choosing the appropriate ABMS tool, when designing and developing their models and prototype systems.

ABMS tools are increasingly used to simulate dynamic small, medium, and large, complex systems and to observe the resulting behaviours in many scientific disciplines such as ecology, climate change, economics, biology, agriculture, sociology, social sciences and STEM disciplines (science, technology, engineering and mathematics). Complex systems can be thought of simply as sets of interacting agents or entities that can represent and mimic organisms, humans, companies, institutions, and any other entity that intends to pursue a particular goal. Agent-based models are particularly applicable in the case of modelling complex phenomena, where many agents or active entities interact with certain inherent attributes to establish interrelationships, facilitating automated thinking and problem solving (Robertson, 2005; North \& Macal, 2007).

Some of the most common areas of application of ABMS tools include (Abar, 2017):

- Cellular automata, Complex adaptive systems, Emergent complex phenomena in Biology / Medical sciences, Epidemiology, Artificial life (Evolutionary computation or genetic programming, Artificial intelligence, Neural networks, Robotics)

- Social \& natural sciences, Dynamic computational systems, Business, Marketing, Economics, Ecology, Healthcare, Planning \& Scheduling, Enterprise and organizational behaviour, Traffic situations (avoidance of traffic jams, light control, route choice)

- Education / Teaching

- Cloud computing / Virtualised datacentres

- Aviation, Flight or air-traffic control, Ground transportation / Mobility planning systems

- Consumer products, Manufacturing, Production (factory based optimized plans for different requirements), Logistics / Distribution / Supply Chains (coordination, storage layout optimization)

- Urban planning (accessibility studies with dynamic populations) 
- Microscopic pedestrian crowd or mapping passenger flow (market improvement \& evacuation of buildings)

- Political phenomena

- Military-combat / Warfighting / Air-defence scenarios

- Financial market's stocks / Securities, Macroeconomic activity

- Large-scale parallel / Distributed computing clusters \& high-performance supercomputers

\section{Conclusion}

The increasing use of ICT in all aspects of life as well as the need to optimize different systems and processes using computer models and simulations, in which intelligent agents play a key role, justify the use of ABMS tools in education, to give future experts an insight into the possibilities of these software solutions.

If we decide to use simulation and ABMS tools in the research, we must consider several key characteristics that will determine the success of the application of this modern technology in each specific case.

One category is the type of software tool license. This feature is of great importance due to the financial aspect of organizing classes.

Other important categories are the programming language for system modelling and simulation, as well as the tool development environment. Getting acquainted with a programming language with which we had no contact until then would be timeconsuming. A complicated development environment would also require too much time to adapt to the tool itself.

We also paid attention to the platforms that support the operation of the tools of this group, as well as to the required operating system. These characteristics represent another significant item from an organizational standpoint.

As a final feature, we will highlight the level of complexity of model development. This feature also has a significant impact on the convenience of choosing someone to model and simulate agent-based tools in the research process.

Mentioned surveys can serve as a useful catalogue in selecting a suitable ABMS tool.

\section{References}

Abar, S., Theodoropoulos, G. K., Lemarinier, P., \& O'Hare, G. M. (2017). Agent Based Modelling and Simulation Tools: A Review of the State-of-Art Software. Computer Science Review, 24, 13-33.

Allan, R. J. (2010). Survey of Agent Based Modelling and Simulation Tools (Technical report, ISSN 1362-0207). Science \& Technology Facilities Council, Warrington, UK. Banks, J., Carson, I. I., Nelson, B. L., \& Nicol, D. M. (2005). Discrete-Event System Simulation. Harlow, UK: Pearson. 
Castle, C. J., \& Crooks, A. T. (2006). Principles and Concepts of Agent-Based Modelling for Developing Geospatial Simulations (Working paper No. 110). Centre for Advanced Spatial Analysis, University College London, London, UK.

Checkland, P. (1981). Systems Thinking, Systems Practice. Chichester, UK: John Wiley \& Sons.

Coyle, R. G. (1996). System Dynamics Modelling: A Practical Approach. London, UK: Chapman \& Hall.

S. Franklin, S. \& Graesser, A. C. (1996). Is it an Agent, or just a Program? A Taxonomy for Autonomous Agents, In Müller, J. P., Wooldridge, M. J., \& Jennings, N. R. (Eds.) Intelligent agent III: Agent Theories, Architectures, and Languages ECAI'96 Workshop, (pp. 21-35). Budapest, Hungary: Springer.

Law, A. M., \& Kelton, W. D. (2000). Simulation Modeling and Analysis (3rd Ed.). New York, NY: McGraw-Hill.

Nikolai, C., \& Madey, G. (2009). Tools of the Trade: A Survey of Various Agent Based Modeling Platforms. Journal of Artificial Societies and Social Simulation, 12(2).

North, M. J., \& Macal, C. M. (2007). Managing business complexity: discovering strategic solutions with agent-based modeling and simulation. Oxford University Press. Pidd, M. (2004). Computer Simulation in Management Science (5th Ed.). Chichester, UK: John Wiley \& Sons.

Pidd, M. (2009). Tools for Thinking: Modelling in Management Science (3rd Ed.). Chichester, UK: John Wiley \& Sons.

Railsback, S. F., Lytinen, S. L., \& Jackson, S. K. (2006). Agent-Based Simulation Platforms: Review and Development Recommendations. Simulation, 82(9), 609-623.

Rakić, K. (2020). Model of Teaching Agent Paradigm using Simulation Environments, Doctoral Dissertation, University of Split, Faculty of science.

Rakić, K., Rosić, M., \& Boljat, I. (2020). A Survey of Agent-Based Modelling and Simulation Tools for Educational Purpose. Tehnički vjesnik, 27(3), 1014-1020.

Robertson, A. (2005). Agent-Based Modeling Toolkits NetLogo, RePast, and Swarm. Academy of Management Learning \& Education 4 (2005) pp. 525-527.

Robinson, S. (2004). Simulation: The Practice of Model Development and Use. Chichester, UK: John Wiley \& Sons.

Russell, S. J., \& Norvig, P. (2003). Artificial Intelligence: A Modern Approach, New Jersey, NJ: Prentice Hall.

Serenko, A., \& Detlor, B. (2002). Agent Toolkits: A General Overview of the Market and an Assessment of Instructor Satisfaction with Utilizing Toolkits in the Classroom (Working paper No. 445). School of Business, McMaster University, Hamilton, CA.

Sokolowski, J. A., \& Banks, C. M. (Eds.). (2009). Principles of Modeling and Simulation: A Multidisciplinary Approach. Chichester, UK: John Wiley \& Sons. Tobias, R., \& Hofmann, C. (2004). Evaluation of Free Java-Libraries for SocialScientific Agent Based Simulation. Journal of Artificial Societies and Social Simulation, 7(1). 